\title{
TRANSVERSE DAMPING SYSTEMS FOR THE FUTURE CERN LHC
}

\author{
E. Gorbatchev, I. Ivanov, V. Kossoukhine, N. Lebedev, V. Melnikov, S. Rabtsoun, JINR, Dubna, Russia \\ W. Höfle*, T. Linnecar, R. Louwerse, J. Tückmantel, CERN, Geneva, Switzerland
}

Abstract

Injection of the high intensity proton beam into the CERN Large Hadron Collider (LHC) presents challenging requirements for the transverse damping system built as a joint project between CERN and JINR (Dubna). This system must quickly reduce injection errors to minimise beam loss to remove risk of quench, and preserve emittance for high luminosity. It should work in a wide frequency range of $20 \mathrm{MHz}$ to fight transverse coupled bunch instabilities. A low frequency electrostatic kicker with tetrode amplifiers has been chosen and the design considerations leading to this choice are described. The kicker design took account of the heating due to the electron cloud effect. A model to simulate the kicker/amplifier combination is presented. Finally preliminary results on the performance of the prototype damper power amplifier and kicker are given.

\section{INTRODUCTION}

In the LHC we require a strong transverse feedback system which combines the functions of feedback (stabilisation against coupled bunch instabilities) and injection damping $[1,2]$. There are three principle tasks for the system

- damping of injection errors

- feedback: curing transverse coupled bunch instabilities (dipole modes)

- excitation of transverse oscillations for beam measurements.

Injection damping requires a powerful system with a bandwidth limited to the ripple frequency of the injection system. In contrast to this, the feedback mode demands a total bandwidth of $20 \mathrm{MHz}$ in order to stabilise all possible coupled bunch dipole modes at the standard bunch spacing of $25 \mathrm{~ns}$. Measurement applications ask for a high flexibility of the system, projected to evolve during the life-cycle of the LHC.

\section{DESIGN PARAMETERS}

Table 1 summarises the principle design parameters [3, 4]. An electro-static system has been chosen. It can deliver the required kick strength at low frequency with a good power efficiency. The planned system is similar in conception to the SPS transverse damper [5]. Note that there are four separate systems, one per plane and beam.

The total required deflecting length of $6 \mathrm{~m}$ is divided into 4 kickers in order to limit the capacitive loading of the

\footnotetext{
*Email: Wolfgang.Hofle @ cern.ch
}

Table 1: Beam Parameters and Requirements

\begin{tabular}{|c|c|c|}
\hline Injection beam momentum & 450 & $\mathrm{GeV} / \mathrm{c}$ \\
\hline Static injection errors $(\beta=183 \mathrm{~m})$ & 2 & $\mathrm{~mm}$ \\
\hline ripple $(\beta=183 \mathrm{~m})$ & 2 & $\mathrm{~mm}$ \\
\hline resistive wall growth time & 14 & $\mathrm{~ms}$ \\
\hline decoherence time & 68 & $\mathrm{~ms}$ \\
\hline tolerable emittance growth & 2.5 & $\%$ \\
\hline overall damping time & 4.7 & $\mathrm{~ms}$ (53 turns) \\
\hline standard bunch spacing & 25 & 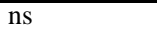 \\
\hline minimum gap between batches & 995 & ns \\
\hline lowest betatron frequency & $>2$ & $\mathrm{kHz}$ \\
\hline highest frequency to damp & 20 & $\mathrm{MHz}$ \\
\hline Electro-static kickers & \multicolumn{2}{|c|}{ base band } \\
\hline aperture of kickers & 52 & $\mathrm{~mm}$ \\
\hline number of kickers per plane and beam & 4 & \\
\hline length of kicker plates & 1.5 & $\mathrm{~m}$ \\
\hline nominal voltage up to $1 \mathrm{MHz}$ & \pm 7.5 & $\mathrm{kV}$ \\
\hline $\begin{array}{l}\text { kick per turn at } 450 \mathrm{GeV} / \mathrm{c} \\
\text { up to } 1 \mathrm{MHz}\end{array}$ & 2 & $\mu \mathrm{rad}$ \\
\hline rise-time $10-90 \%, \Delta V= \pm 7.5 \mathrm{kV}$ & 350 & ns \\
\hline rise-time $1-99 \%, \Delta V= \pm 7.5 \mathrm{kV}$ & 720 & ns \\
\hline frequency range for gain & $0.001-20$ & $\mathrm{MHz}$ \\
\hline $\begin{array}{l}\text { noise dominated by } \\
\text { pick-up signal quantisation }\end{array}$ & \multicolumn{2}{|c|}{$10 \mathrm{bit} / 2 \sigma_{\text {beam }}$} \\
\hline
\end{tabular}

power amplifiers. The maximum kick per turn is $2 \mu \mathrm{rad}$ at $450 \mathrm{GeV} / \mathrm{c}$, the location of the kickers is foreseen at $\beta \geq 100 \mathrm{~m}$. At injection the resulting emittance blow-up after filamentation and damping depends very critically on the assumed ratio of decoherence time and damping time. Values given in Table 1 are calculated using simple formulae to estimate the blow-up [6]. A faster rise time of the resistive wall instability, possible due to a higher machine impedance, reduces the safety margin, but could be compensated by additional damping systems.

The kicker elements are installed on either side of IP4 in the RF zones at locations with high beta functions. Power amplifiers and kickers are being developed and built in collaboration with the JINR in Dubna, Russia. The first prototype models of amplifier and kicker have fulfilled the specified key parameters of bandwidth and kick strength.

\section{SYSTEM OVERVIEW}

Fig. 1 shows a block diagram with the principle elements of the system. Two coupler type pick-ups with an electrode length of $150 \mathrm{~mm}$ will be dedicated to each damper system. Combining the signals from the pick-ups allows adjustment of the feedback phase for optimum damping and stability. Between pick-ups and ADC the signal levels must be adjusted for optimised performance (dynamic range). The digital filtering itself will contain processing for closed or- 


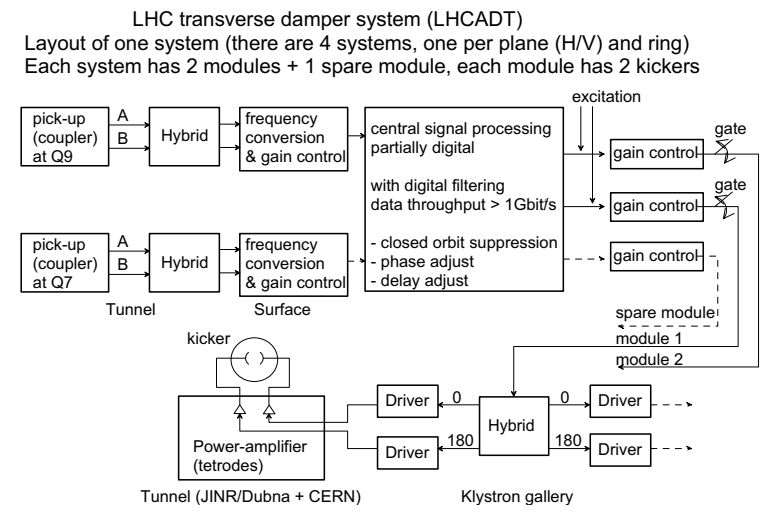

Figure 1: Block diagram of the LHC damper system.

bit suppression, delay adjustment, gating, and bunch synchronised input for beam measurement purposes (e.g. tune measurement). The pick-up signals are digitised so that the ADC of 10 bits corresponds to a beam movement of $2 \times$ the rms beam size. For ultimate low noise performance it might be required to center the beam in the physical apertures of the pick-ups. This could be achieved by a slow feedback on the orbit correction system.

The overall feedback gain in the loop will be tuned after the digital processing by a function controlled amplifier. During injection we require a high gain for rapid damping, while during the ramp and storage the gain should be kept low to minimise blow-up due to damper noise.

Underground areas will not be accessible when beams are circulating. Therfore the low level signal processing will be installed on the surface. The driver amplifiers $(\simeq 170 \mathrm{~W}$ in $50 \Omega$ ) can be located in an auxiliary tunnel underground ("klystron gallery") and the power amplifiers with tetrodes will be located under the kicker tank in the main tunnel. Note that the distributed nature of the installation with cabling distances of about $600 \mathrm{~m}$ between the underground area and the surface service buildings requires a high degree of remote monitoring.

\section{KICKER DESIGN}

The kickers for the transverse feedback system will be installed in the RF insertion of the LHC in point 4 . The exact location is chosen for large beta functions in order to maximize the efficiency of the kickers.

\subsection{Mechanical design}

The different requirements for vacuum, RF electrical properties and alignment accuracy have led to the mechanical design depicted in Fig. 2. It shows a transverse and longitudinal section through a vertical kicker. As they form part of the room-temperature vacuum system of the LHC, a tank with $100 \mathrm{~mm}$ diameter (1) has been chosen to house the deflecting electrodes (2). The tank is machined from stainless steel providing a solid, mechanically stable construction which allows very accurate positioning. The wall thickness of $14 \mathrm{~mm}$ provides adequate shielding at $3 \mathrm{kHz}$, the lowest betatron frequency, under all circumstances.

The electrodes (2) are shaped from copper strips as $90^{\circ}$ arcs leaving an aperture of $52 \mathrm{~mm}$ for the beam. The two opposing electrodes are brazed onto three ceramic rings (3) which provide mechanical support and electrical insulation. The rigid electrode assembly is centred inside the vacuum tank by means of 6 rollers. Spring contacts and a central locking post (4) prevent longitudinal and rotational movement. The electrical contacts are located in the center. Flexible pins made of copper connect to the two ceramic vacuum feed throughs (5) which are located in the horizontal planes, for both the horizontal and vertical kicker types.

\subsection{Heating of electrodes by beam image cur- rent and electron cloud effect}

Heating of the electrodes in vacuum was identified as important early on in the design and has become critical due to the additional heat load produced by the electron cloud effect during commissioning of the LHC [7]. The heat load on the electrodes by the beam image current alone is estimated to be $2 \mathrm{~W} / \mathrm{m}$ per electrode for the ultimate beam current $\left(1.7 \times 10^{11}\right.$ protons per bunch). Calculations showed that considering only cooling by radiation would yield an unacceptably high temperature rise of over $100 \mathrm{~K}$ on the electrodes. In order to estimate correctly the additional cooling by means of the electrical connection (feed through) and the mechanical supports (ceramic rings) tests on a prototype kicker were performed. $2-5 \mathrm{~W} / \mathrm{m}$ per electrode was dissipated uniformly along the electrodes and the temperature increase under vacuum measured. The equilibrium was reached after 20-30 hours, the temperature increase varying between $22 \mathrm{~K}$ for $2 \mathrm{~W} / \mathrm{m}$ per electrode and $47 \mathrm{~K}$ for $5 \mathrm{~W} / \mathrm{m}$. These temperature rises are acceptable and would allow for an additional heating by the electron cloud effect of $3 \mathrm{~W} / \mathrm{m}$ per electrode. In the final design the tolerable heat load will be higher due to increased cooling by a better thermal contact via the feedthrough.

\section{POWER AMPLIFIER - SIMULATION AND TESTS}

Fig. 3 shows the simplified electrical circuit of the prototype power amplifier. Two vacuum tetrodes Siemens RS2048 (CJ) work in push-pull mode on the kicker which presents a capacitive load at low frequency. The parasitic capacitance of the connection between amplifier and kicker is reduced by keeping the distance between feedthroughs and tetrodes as short as possible. The best high frequency behaviour is achieved by matching the impedance of the connecting line to the kicker impedances. Together with the parasitic inductance (feed-through and connection inside the vacuum tank) the transmission line characteristics can be well approximated by series inductances $(345 \mathrm{nH})$ as shown in Fig. 3 .

The electrical circuit was modelled using Microsim 


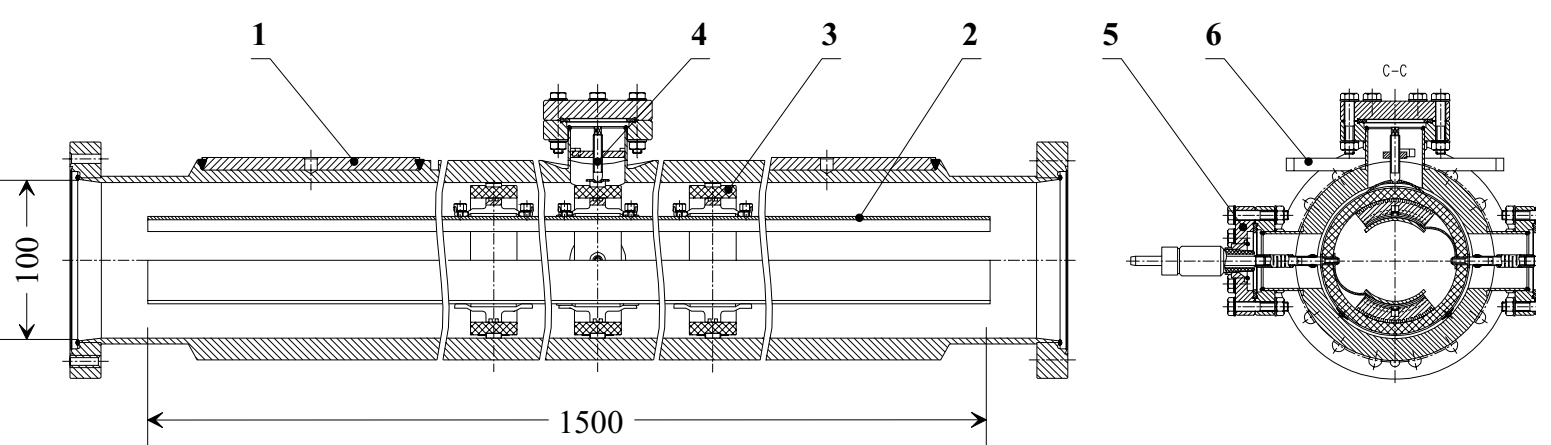

Figure 2: Mechanical design of kicker (vertical), vacuum tank (1), electrodes (2), ceramic rings (3), central locking post (4), feed through (5), reference plate for alignment (6).

PSpice $\left.{ }^{(}\right)$which allows full simulation of the non-linear regimes including DC, AC (frequency domain) and transient (time domain) analyses. The amplifier works in a nonlinear regime, where the usual simple method of linearisation of the tetrode characteristics do not give acceptable results. A model of the tetrode has been developed which takes into account the dependence of the anode and screen grid currents on the anode, screen grid, and control grid voltages [8]. The control grid current is assumed to depend only on the control grid voltage. The quality of the simulation at high frequency depends critically on the knowledge of all parasitic elements in the circuit, such as mounting capacities and inductances of connections. An amplifier and

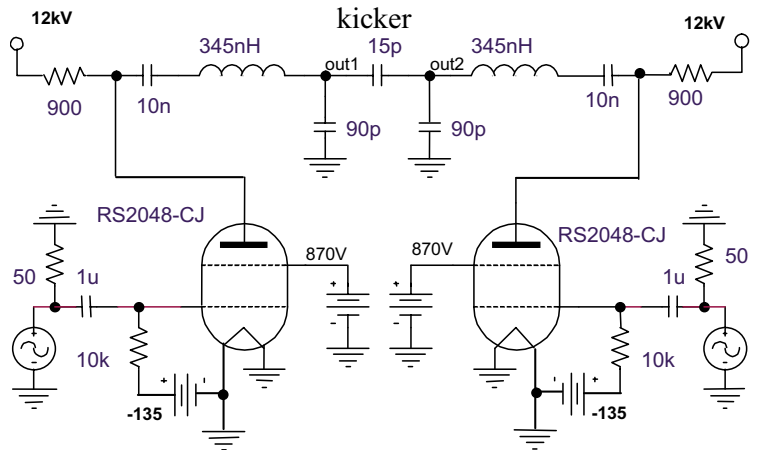

Figure 3: Simplified electrical circuit of the amplifier.

kicker prototype were built and tested in the lab. Simulated and measured gain and phase response are shown in Fig. 4. The agreement is very good up to $20 \mathrm{MHz}$ [8], which will be the maximum operating frequency. The phase characteristics can be linearized in the low level part of the feedback. The experimental results on the kicker and amplifier prototype assembly verify the design principle. Work has started towards final design approval and series production preparation. The current project plan foresees delivery by JINR to CERN of the power amplifiers and kickers by end of 2003.

\section{ACKNOWLEDGEMENTS}

The work on the power amplifiers and kickers for the transverse dampers is carried out under the 1992 collaboration agreement between CERN, Geneva, Switzerland,
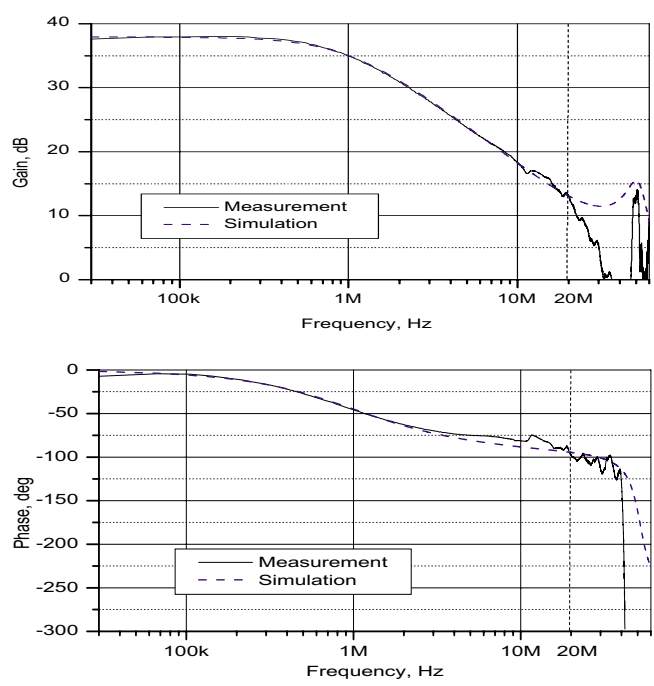

Figure 4: Simulated and measured gain and phase response of the power amplifier kicker assembly.

and the Joint Institute for Nuclear Research (JINR), Dubna, Russia. It represents part of the contribution of the JINR to the LHC project at CERN.

\section{REFERENCES}

[1] P. Lefèvre, T. Pettersson (eds.) "The Large Hadron Collider", Conceptual Design, CERN/AC/95-05 (LHC), Geneva, 1995.

[2] D. Brandt, Workshop on LEP-SPS Performance, CERN-SL2000-007 (DI), Geneva, 2000, pp. 178-181.

[3] D. Boussard, W. Höfle, T. Linnecar, CERN SL-Note-99-055 (HRF), 1999.

[4] W. Höfle, LHC Workshop, CERN-SL-2001-003 (DI), Geneva, 2001, pp. 317-319.

[5] W. Höfle, Particle Accelerators, 58, 1997, pp. 269-279.

[6] L. Vos, $6^{\text {th }}$ European Particle Accelerator Conference, EPAC, Stockholm, June 22-26 1998, 1365-1367.

[7] The latest news can be found under the URL: http://wwwslap.cern.ch/collective/electron-cloud/electroncloud.html

[8] E. Gorbatchev, V. Melnikov, W. Höfle, LHC-Project-Note259, CERN, Geneva 2001 